\title{
Development of a debutanizer column soft sensor by means of evolutionary machine learning approaches
}

\author{
Matheus Henrique Reis Marchioro*, Victor Henrique Alves Ribeiro**, Gilberto Reynoso-Meza** \\ *Department of Control and Automation Engineering, Pontifícia Universidade Católica do Paraná, Curitiba, Paraná, \\ Brazil (e-mail: matheus.mhrm@gmail.com). \\ **Industrial and Systems Engineering Graduate Program, Pontifícia Universidade Católica do Paraná, Curitiba, Paraná, \\ Brazil (e-mail: victor.henrique@pucpr.edu.br,g.reynosomeza@pucpr.br)
}

\begin{abstract}
Soft sensors have been widely employed to predict variables from a process that cannot be measured. In literature, there are many techniques that can be used to develop a soft sensor. The robustness of the model is directly related to the complexity of the techniques employed in the model. However, it does not mean that simple models are not able to achieve good results. Support Vector Machines (SVM) and Decision Trees (DT) has been shown as good candidates to build soft sensors. Two experiments are proposed in this work. First, three benchmark regression data sets are studied. Finally, a debutanizer column is provided as study case in order to build a soft sensor. Using Genetic Algorithm (GA) as a tool to improve the developed models, it is possible to perceive that there are no significant differences between the results achieved with SVM and DT in terms of RMSE. However, analyzing the aspects of the predicted curves, the results obtained with SVM is the closest to the actual data.
\end{abstract}

Resumo: Sensores virtuais têm sido amplamente empregados para predição de variáveis de um processo que não podem ser mensuradas. Na literatura, existem diversas técnicas que podem ser usadas para desenvolver um sensor virtual. A robustez do modelo está diretamente relacionada à complexidade das técnicas empregadas no modelo. No entanto, isso não significa que modelos simples não sejam capazes de alcançar bons resultados. As Máquinas de vetores de suporte (SVM) e as Árvores de Decisão (DT) se apresentaram como boas candidatas para criação de soft sensors. Dois experimentos são propostos neste trabalho. Primeiramente, três conjuntos de dados de regressão são estudados. Finalmente, uma coluna debutanizadora é fornecida como caso de estudo para construir um sensor soft. Utilizando o Algoritmo Genético (GA) como ferramenta para melhorar os modelos desenvolvidos, é possível perceber que não há diferenças significativas entre os resultados obtidos com SVM e DT em termos de RMSE. No entanto, analisando os aspectos das curvas previstas, o resultado obtido com o SVM é o mais próximo dos dados reais.

Keywords: Soft Sensors; Machine Learning; Evolutionary Computing; Support Vector Machine; Decision Tree; Genetic Algorithm.

Palavras-chaves: Sensores Virtuais, Aprendizado de Máquina, Computação Evolucionária, Máquinas de Vetor de Suporte, Árvores de Decisão, Algoritmo Genético.

\section{INTRODUCTION}

In order to maintain a desired quality, every control or monitoring system requires the use of interfaces with the physical world. Thus, industrial processes must be well equipped with a diversity of sensor to be able to monitor their current state and take decisions. However, there are situations where not all variables from a process can be measured. It can happen for several reasons, such as situations that requires real-time information of the process and the proper sensor does not exist or is relatively expensive. Therefore, many researchers started using available information from the process to create predictive models as way of estimate the value of "hard-to-measure" variables. In the literature, these models are known as Soft Sensors.
In agreement with Kadlec et al. (2009) there are two types of soft sensors, the model-driven and data-driven. The first model is based on the process descriptions, that is, the system has the complete knowledge of the phenomenon that governs the process. Data-driven models, on the other hand, are based on the empirical observations of the process, and have no knowledge on the process. Although data-driven models, unlike the model-driven, is a black-box model, they are better at describing the true conditions of the process than the model-driven.

One possible solution to create such systems is using supervised machine learning which, different from traditional programming, uses available input and output data to develop prediction models. Machine learning consists of a field of 
computer science, which aims to explore the study and construction of algorithms that give to machines the ability to learn and predict data without being previously programmed to do it.

This project focuses in using evolutionary optimization to perform model selection on the creation of prediction models for soft sensor development. Section 2 brings the fundamentals. Sections 3 and 4 details, respectively, the materials and methods adopted for the design of the project and the experimental results obtained, while the discussion and conclusion are presented in sections 5 and 6 .

\section{FUNDAMENTALS}

\subsection{Machine Learning}

According to Domingos (2015), the main rival schools of thought that exist within machine learning are the Symbolists, Connectionists, Bayesians, Analogizers, and Evolutionaries, which have a set of core beliefs, and a particular problem that it cares most about. These schools will be briefly described below.

For Symbolists, all intelligence can be reduced to manipulating symbols, in the same way that a mathematician solves equations by replacing expressions by other expressions (Domingos, 2015). Basically, Symbolists understand that you need some initial knowledge to work with data. Their algorithms work with the idea of incorporating the preexisting knowledge with the learning, aiming to join each piece of knowledge in order to solve new problems. As representative of the Symbolists, we have the Decision Trees (DT). This algorithm uses a tree-like model of decisions and their consequences, that starting at the "root", each node has two branches and depending on the choice, it's possible to follow a specific branch. At the end, a prediction can be obtained.

Domingos (2015) defines that, according to Connectionists, learning consists exactly in the same process that the human brain does to acquire knowledge. The greatest representative of this school consists in Artificial Neural Networks (ANN). Even though several models have been developed over the years, each of them follows the same idea: Knowledge is acquired by the network through a learning process, which is stored in the forces of connections between neurons, known as "synaptic weights" (Haykin, 2003).

Bayesians are concerned above all with uncertainty. All learned knowledge is uncertain, and learning itself is a form of uncertain inference (Domingos, 2015). The problem addressed by this school is how to deal with the uncertainty and incompleteness of information. Their solution includes the implementation of an algorithm based on the Bayes' theorem. This model, in a manner similar to DT, works with a tree model, however the path to be followed is determined by probabilities.

Recognition of patterns between situations and their application in similar situations can be defined as the principle that governs the entire Analytic school. Pursuant to Domingos (2015), the Analogizers' master algorithm is Support Vector Machine (SVM). Ribeiro (2017) defines SVM as an algorithm that can solve linear and nonlinear classification problems by means of a hyper plane, which is optimized from the available data.

Evolutionaries believe that the mother of all learning is natural selection. If it made us, it can make anything, and all we need to do is simulate it on the computer (Domingos, 2015). In agreement with him, the main problem that this strand solves is optimization of learning structure. Their master algorithm is genetic programming, which computer programs are encoded as set of genes that evolves, in the same way that nature works, using an evolutionary algorithm, which will be discussed below.

\subsection{Optimization of Machine Learning Models}

As previously stated, evolutionary programs in general aims to optimize learning structures. Seeking to achieve this goal with predictive models, there are three types of optimization problems in the literature that have can be performed, which are: Feature selection (FS), Instance Selection (IS) and Hyper Parameter Selection (HS). According to Perez et al. (2017), one of the major steps in data mining process consists in data preprocessing, which can have a significant impact on the performance of a machine learning algorithm.

In data mining and machine learning, real-world problems often involve a large number of features. However, not all features are essential since many of them are redundant or even irrelevant, which may reduce the performance of an algorithm, e.g., a classification algorithm (Xue et al., 2016).

The same idea can be applied to instances, since in most part of the times the quality of dataset is not high, hence the incompleteness of information, as well as incorrect information may too reduce the performance of an algorithm. One of the most influential data preprocessing is the instance selection. IS aims at selecting a relevant instance subset of the entire training set, while preserving the performance of the whole dataset (Perez et al., 2017). In this context, evolutionary techniques have been widely used as form to remove irrelevant features and erroneous instances.

Depending on the complexity of the algorithm to be used in the optimization, there may be hyper parameters that, when altered, influence the quality of the result. Aydin et al. (2011) proposed a multi-objective artificial immune algorithm for parameter $C$ and $\sigma$ optimization in SVM aiming to increase accuracy of this model. This is a good example to understand the operation of HS, however this model will not be implemented in this project.

\section{METHODOLOGY}

\subsection{Support Vector Machine}

The development of SVMs has a 30-year history, from 1965 until 1995 (Vapnik, 2006). The original algorithm 
constructed by Vladimir N. Vapnik in 1965 was a linear classifier. Only in 1995 Vapnik et al. suggest a way to create non-linear classifier applying the kernel method proposed by Aizerman et al. in 1964.

In short, Support Vector Machine consists in a supervised learning algorithm which can be used for classification, regression or other tasks like outlier detection solving linear and non-linear problems. In order to solve these problems, SVM constructs a hyperplane or set of hyperplanes in a high or infinite dimensional and seeks to maximize the distance to the nearest training data point of any class.

In addition to performing in linear problems, SVM can be applied to solve non-linear problems mapping their inputs in high-dimensional feature spaces, where data can be separated linearly, such process is called as "kernel trick". The resulting algorithm is formally similar, except that every dot product is replaced by a non-linear kernel function. The common kernel functions are: Polynomial (homogenous), Polynomial (inhomogeneous), Gaussian and Hyperbolic tangent.

There are only a few minor differences when working with regression problems, however the principles used in SVM for classification problems are still the same. Since the output is a real number, it becomes very difficult to predict the information. Aiming to solve this problem, a margin of tolerance (epsilon) is set in approximation to the SVM which would have already requested from the problem. In the literature this technique is also known as Support Vector Regression (SVR).

\subsection{Decision Tree}

According to Breiman (1984), decision Tree builds regression or classification models in the form of a tree structure. Basically, this technique breaks down a dataset into a smaller subset while at the same time a new tree is developed from the subset. The final result is a tree with decision nodes and leaf nodes. A decision node has two or more branches, each representing values for the attribute tested. Leaf node represents a decision on the numerical target.

In order to find solutions DT makes sequential, hierarchical decision about the outcomes variable based on the predictor data. In other words, every DT model is made by a series of questions that when answered, lead to a class label or a value.

\subsection{Genetic Algorithm}

Even though in the literature of evolutionary computing there are several algorithms, there's none better than other, each of them has their purpose. However, for this project it will be used Genetic Algorithm (GA).

According to Mitchell (1999), GA is a method developed by John Holland and his students inspired in the phenomenon of natural adaptation as it occurs, and how it could be imported to a computer. In short, GA is a method for moving from one population of "chromosomes" to a new population mimicking the nature. Each chromosome consists of "genes' (bits), each gene being an instance of "allele" ( 0 or 1$)$. There are some operators called Selection, Crossover and Mutation, to allow this to occur. The selection operator chooses some chromosomes that will be allowed to reproduce. Crossover is responsible to combine two chromosomes and create a third. Lastly, Mutation is the operator that can change the chromosomes randomly.

\subsection{Benchmark Regression Problems}

In order to acquire the necessary knowledge for the development of the project, with MATLAB support, several implementations involving the following algorithms were performed: Support Vector Machine and Decision Tree, involving regression and classification problems from UCI Machine Learning Repository. Then, optimization tests were performed using the genetic algorithm for the feature selection, instance selection and hyper parameters selection cases, aiming, at different moments, to minimize a specific error metric, being: Mean Absolute Error (MAE), Root Mean Squared Error (RMSE) and Mean Absolute Percentage Error (MAPE). For this project RMSE was employed in order to performance evaluation. It can be defined as:

$$
\text { RMSE }=\sqrt{\frac{\sum_{i=1}^{n}\left(y_{\text {real }(i)}-y_{\text {predicted }(i))^{2}}\right.}{n}}
$$

Where $\mathrm{n}$ is the number of testing samples, $y_{\text {reallit }}$ is the actual value obtained by measurements, and $y_{\text {predicted }}(\bar{D})$ is the predicted value.

Thus, three UCI data sets involving regression problems were selected, since they present a higher level of difficulty in prediction, as well as being closer to problems involving Soft Sensors. Table 1 presents a brief description of the data sets used.

Table 1. Description of the datasets

\begin{tabular}{|l|l|c|c|}
\hline \multicolumn{1}{|c|}{ Name } & \multicolumn{1}{|c|}{ Objective } & Instances & Features \\
\hline $\begin{array}{l}\text { Airfoil Self- } \\
\text { Noise }\end{array}$ & $\begin{array}{l}\text { Predict Scaled } \\
\text { sound pressure } \\
\text { level (dB) }\end{array}$ & 1503 & 5 \\
\hline $\begin{array}{l}\text { Combined } \\
\text { Cycle } \\
\text { Power Plant }\end{array}$ & $\begin{array}{l}\text { Predict Output } \\
\text { Potency (MW) }\end{array}$ & 9568 & 4 \\
\hline $\begin{array}{l}\text { Energy } \\
\text { Efficiency }\end{array}$ & $\begin{array}{l}\text { Predict Heating } \\
\text { Load }\end{array}$ & 768 & 8 \\
\hline
\end{tabular}

\subsection{Soft Sensor Problem}

The chosen problem for soft-sensor application consist in an industrial process called Debutanizer column, which is composition of distillation process, whose main goal is to remove propane and butane from the naphtha stream. 
Even though in this process it is crucial to detect the content of butane in the bottom of the column for monitoring the process, the measurement of the content is not a simple task, because it takes a long time delay to execute. Thus, a good alternative to work around this problem is to predict the butane content based on other easily-measured variables. There are seven process variables chosen as inputs for softsensor modeling which cover temperatures, pressures and flows similar to that used by Yao and Ge (2017) and Liu et al. (2018). The detailed description of the debutanizer column and process variables is given in Fig 1 and Table 2.

The process has a nonlinear relationship due to several disturbances, which means that a linear model cannot be used for forecasting. Therefore, the Gaussian kernel function was used in Support Vector Machine technique since it has the best capacity to detect nonlinearities.

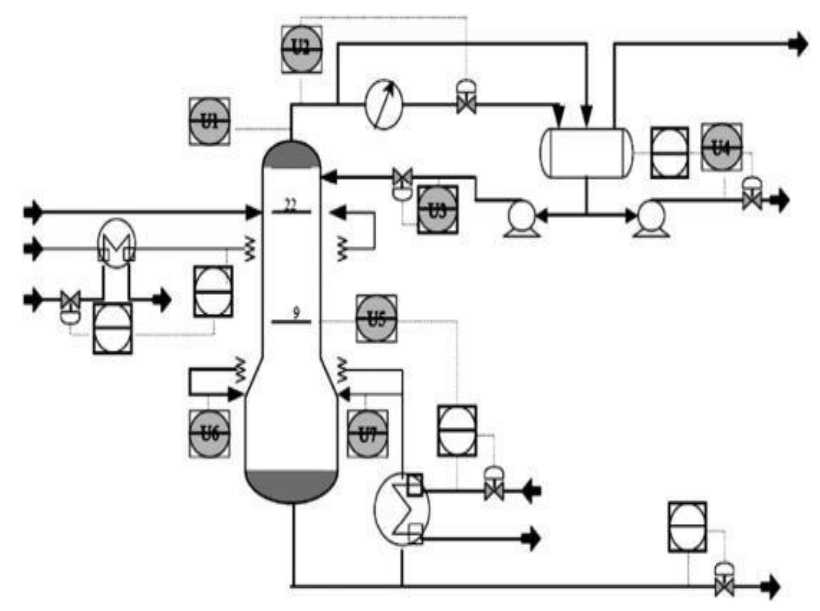

Fig. 1 Flowchart of debutanizer column. (Yao and Ge, 2017)

Table 2. Process Variables. (Yao and Ge, 2017)

\begin{tabular}{|c|l|}
\hline Input variables & \multicolumn{1}{|c|}{ Description } \\
\hline U1 & Top temperature \\
\hline $\mathrm{U} 2$ & Top pressure \\
\hline $\mathrm{U} 3$ & Reflux flow \\
\hline $\mathrm{U} 4$ & Flow to next process \\
\hline $\mathrm{U} 5$ & $6^{\text {th }}$ tray temperature \\
\hline $\mathrm{U} 6$ & Bottom temperature \\
\hline $\mathrm{U} 7$ & Bottom pressure \\
\hline
\end{tabular}

\section{EXPERIMENTAL RESULTS}

\subsection{Benchmark Regression Problems}

The results obtained using the proposed algorithms are evidenced for DT in table 3 and for SVM in table 4 . It is possible to perceive that in Airfoil Self- Noise and Combined Cycle Power Plant datasets presented better results using SVM with instance selection. On the other hands, for Energy Efficiency dataset, the best result obtained was using DT with feature selection.

Table 3. Decision Tree Results (RMSE)

\begin{tabular}{|l|c|c|c|}
\hline \multicolumn{1}{|c|}{ Name } & $\begin{array}{c}\text { Without } \\
\text { Optimization }\end{array}$ & $\begin{array}{c}\text { Instance } \\
\text { Selection }\end{array}$ & $\begin{array}{c}\text { Feature } \\
\text { Selection }\end{array}$ \\
\hline $\begin{array}{l}\text { Airfoil Self- } \\
\text { Noise }\end{array}$ & 6.4115 & 3.3310 & 6.4115 \\
\hline $\begin{array}{l}\text { Combined } \\
\text { Cycle } \\
\text { Power Plant }\end{array}$ & 4.5669 & 4.1885 & 4.1885 \\
\hline $\begin{array}{l}\text { Energy } \\
\text { Efficiency }\end{array}$ & 1.0316 & 0.8583 & 0.8479 \\
\hline
\end{tabular}

Table 4. Support Vector Machine Results (RMSE)

\begin{tabular}{|l|c|c|c|}
\hline \multicolumn{1}{|c|}{ Name } & $\begin{array}{c}\text { Without } \\
\text { Optimization }\end{array}$ & $\begin{array}{c}\text { Instance } \\
\text { Selection }\end{array}$ & $\begin{array}{c}\text { Feature } \\
\text { Selection }\end{array}$ \\
\hline $\begin{array}{l}\text { Airfoil Self- } \\
\text { Noise }\end{array}$ & 3.5571 & 2.7255 & 3.5571 \\
\hline $\begin{array}{l}\text { Combined } \\
\text { Cycle } \\
\text { Power Plant }\end{array}$ & 4.0324 & 3.8926 & 4.0324 \\
\hline $\begin{array}{l}\text { Energy } \\
\text { Efficiency }\end{array}$ & 4.5911 & 2.9482 & 2.5621 \\
\hline
\end{tabular}

The best results for each dataset can be better evidenced in Figures 2, 3 and 4, which presents the predictions for the SVM with instance selection for the Airfoil Self-Noise and Combined Circle Power Plant problems and the DT with feature selection for the Energy Efficiency problem, respectively.

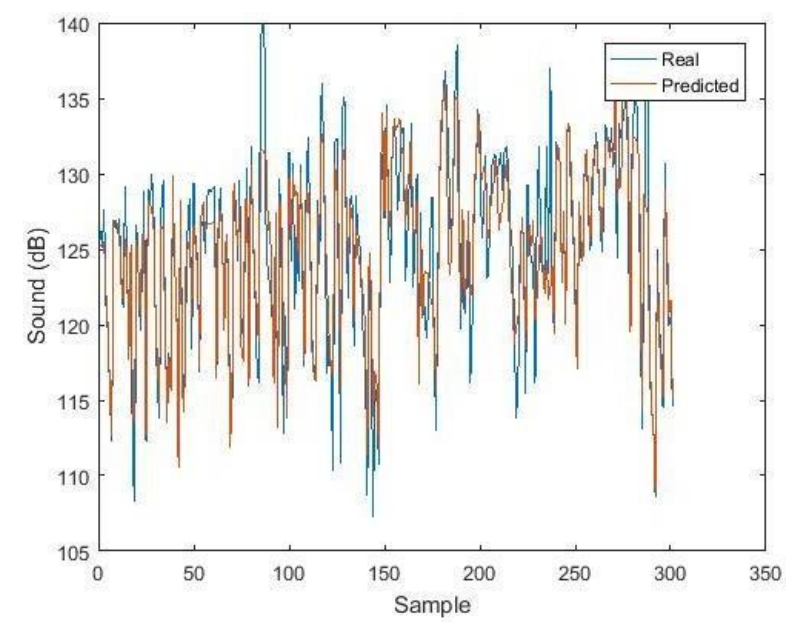

Fig. 2 Airfoil Self-Noise (Best model): Using instance selection with Support Vector Machine (SVM). 


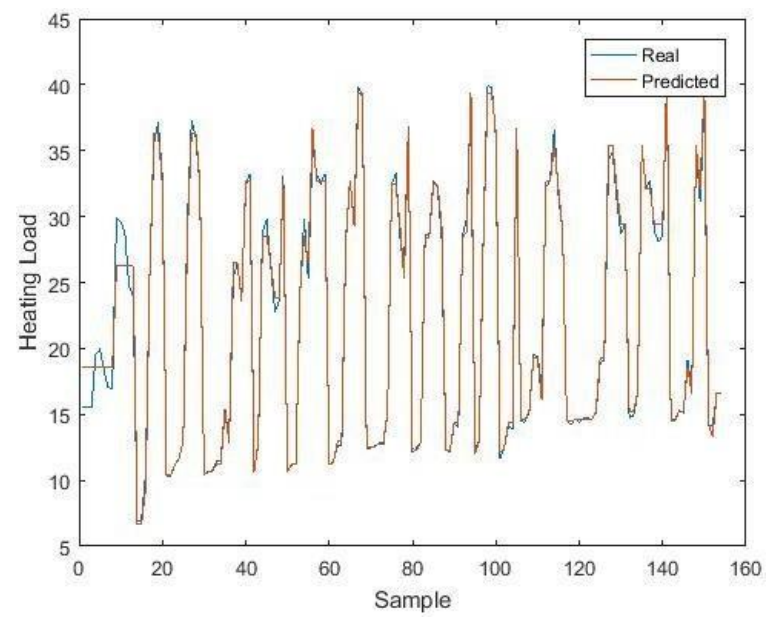

Fig. 3 Combined Cycle Power Plant (Best model): Using instance selection with Support Vector Machine (SVM).

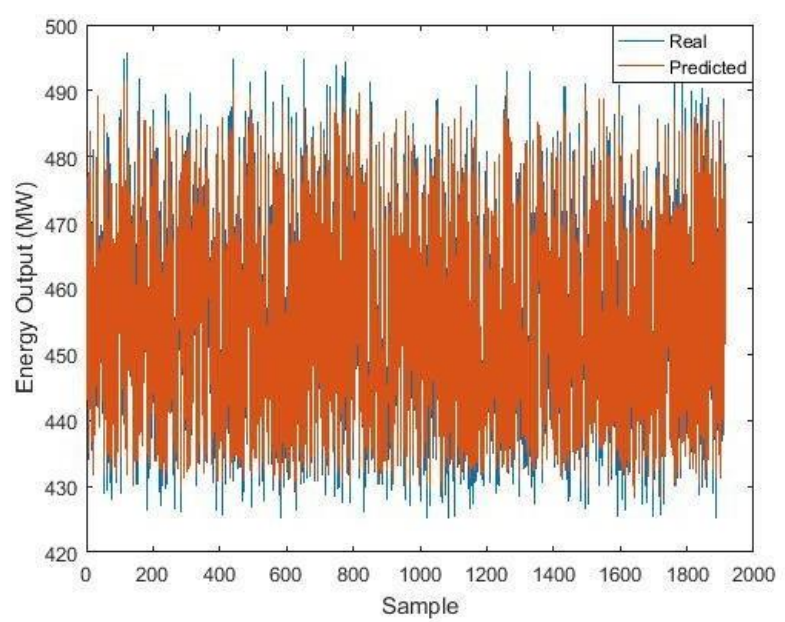

Fig. 4 Energy Efficiency (Best model): Using feature selection with Decision Tree (DT).

\subsection{Soft Sensor Development}

Table 5 and Figures 5, 6, 7 and 8 show the obtained results using Decision Tree and Support Vector Machine algorithms for debutanizer column problem.

Table 5. Debutanizer Column Results (RMSE)

\begin{tabular}{|c|c|c|c|}
\hline Algorithm & $\begin{array}{c}\text { Without } \\
\text { Optimization }\end{array}$ & $\begin{array}{c}\text { Instance } \\
\text { Selection }\end{array}$ & $\begin{array}{c}\text { Feature } \\
\text { Selection }\end{array}$ \\
\hline $\begin{array}{c}\text { Decision } \\
\text { tree }\end{array}$ & 0.1297 & 0.1046 & 0.1250 \\
\hline SVM & 0.1218 & 0.0979 & 0.1010 \\
\hline
\end{tabular}

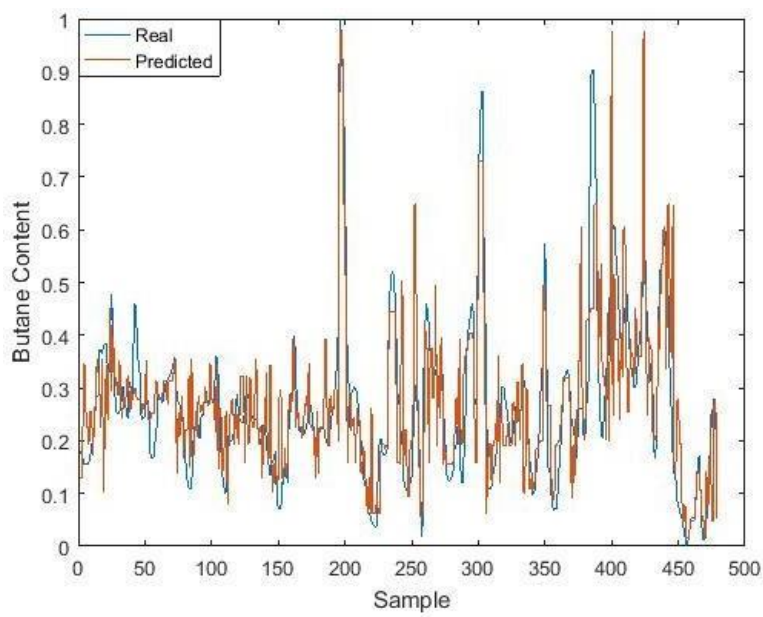

Fig. 5 Debutanizer Column: Using instance selection with Decision Tree (DT).

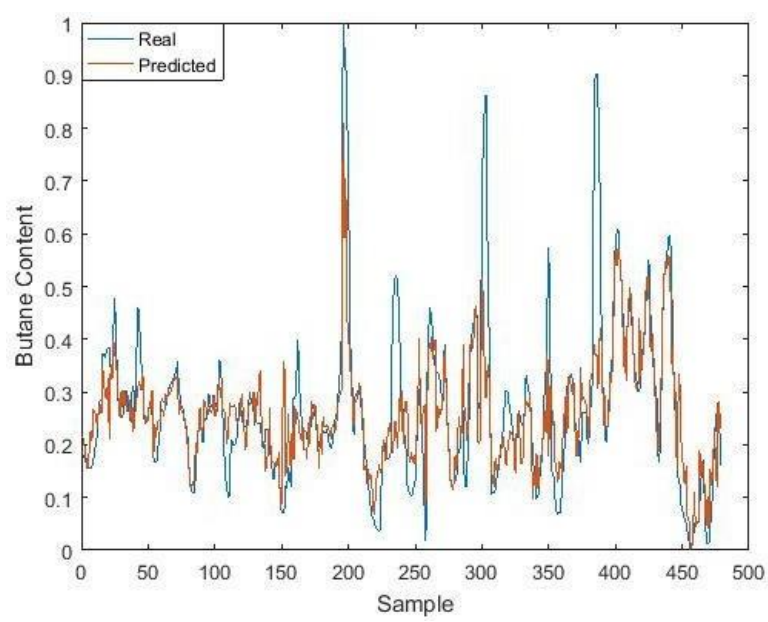

Fig. 6 Debutanizer Column: Using instance selection with Support Vector Machine (SVM).

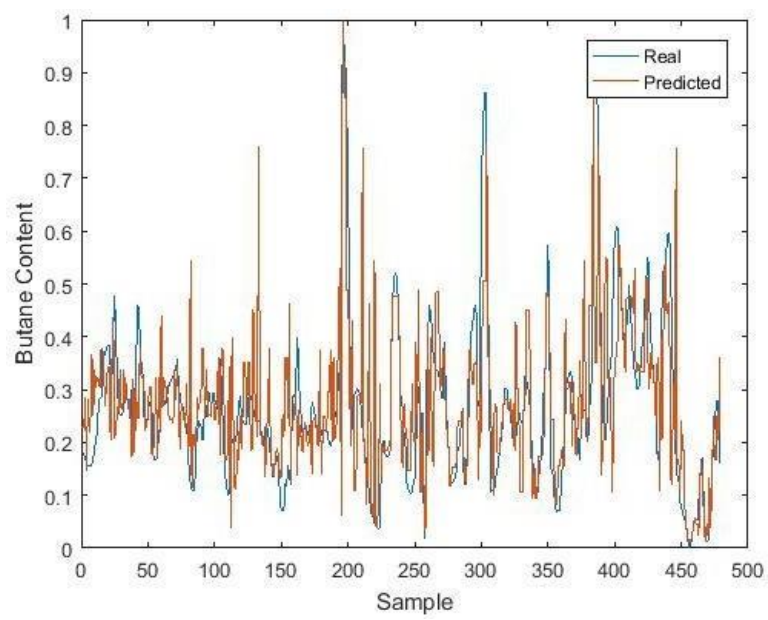

Fig. 7 Debutanizer Column: Using feature selection with Decision Tree (DT). 


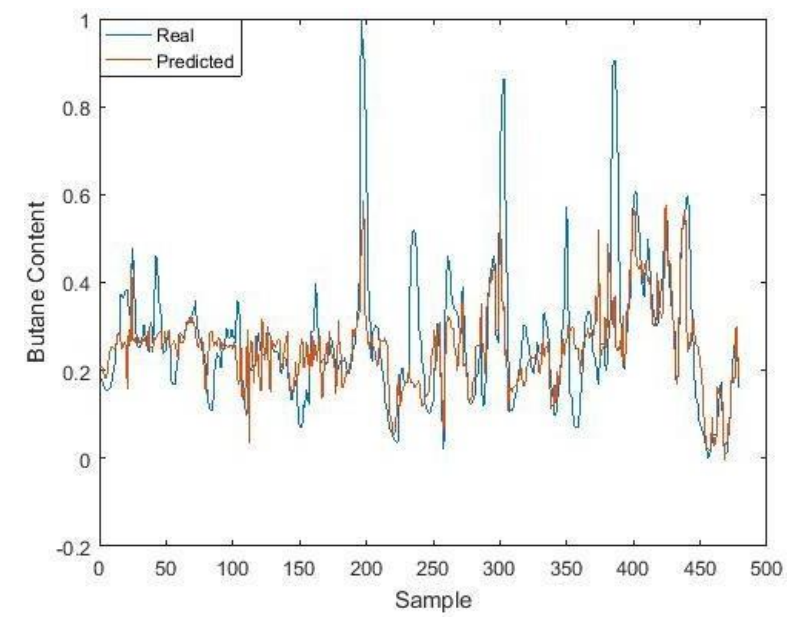

Fig. 8 Debutanizer Column: Using feature selection with Support Vector Machine (SVM).

\section{DISCUSSION}

It is possible to perceive that there is a certain pattern of behavior of the answers obtained using both algorithms. Regarding DT, although the technique is able to predict some non-captured peaks using SVM, the result has a noisier appearance, thus making the prediction in general perform worse, as evidenced by its RMSE value. On the other hand, the SVM technique, although it cannot predict some less common peaks in the process, keeps closer to the real model in general terms. Furthermore, when analyzing Figures 6 and 8 , it is verified a great improvement in the aspect of the prediction using instance selection instead of feature selection for SVM.

\section{CONCLUSIONS}

Even though the results obtained are not better than those found by Yue and Ge (2017) and Liu et al. (2018), they proved to be extremely satisfying since they were achieved using a simple approach, more specifically, a single objective optimization.

It is worth remembering that this work is only a starting point for a larger project, which aims to use computational intelligence techniques to develop robust soft sensors, by means of several engineering benchmark/competition data sets focused in regression and classification problems These data sets will be selected and used in order to analyze the use of multi-objective optimization for machine learning model selection.

\section{AKNOWLEDGMENTS}

This study was financed in part by Pontíficia Universidade Católica do Paraná (PUCPR), Coordenação de Aperfeiçoamento de Pessoal de Nível Superior (CAPES) and onselho Nacional de Pesquisa e Desenvolvimento (CNPq) Brazil - Finance Codes: PP1736829-PIBIC, 159063/2017-0PROSUC, 304066/2016-8-PQ2, 437105/2018-0-Univ and PRONEX-042/2018.

\section{REFERENCES}

Aydin, I., Karakose, M., Akin, E. A multi-objective artificial immune algorithm for parameter optimization in support vector machine. Applied Soft Computing, volume (11), pages $120-129$.

Breiman, L. (2017). Classification and Regression Trees. Routledge. London.

Domingos, P. (2015). The Master Algorithm: How the Quest for the Ultimate learning Machine Will Remake Our World. Basic Books. New York, USA.

Dua, D. and Graff, C. (2019). UCI Machine Learning Repository [http://archive.ics.uci.edu/ml]. Irvine, CA: University of California, School of Information and Computer Science.

Haykin S (2003). Redes Neurais Princípios e Prática. $2^{\mathrm{a}}$ ed. Bookman CIA Editora Ltda. Porto Alegre RS.

Kadlec, P., Gabrys, B., Strandt, S. (2009). Data-driven soft sensors in the process industry. Computers \& chemical engineering, volume (33), pages 795-814

Liu, Z., Ge, Z., Chen, G., Song, Z. (2018). Adaptive soft sensors for quality prediction under the framework of Bayesian network. Control Engineering Practice, volume (72), pages 19-28.

Mitchell, M. (1999) An Introduction to Genetic Algorithms. A Bradford book. London.

Ribeiro, V. Multi-Objective Model Selection for Unmanned Aerial Vehicles Automatic Target Recognition Systems. 116 f. Masters Dissertation - Pontifícia Universidade Católica do Paraná.

Rosales-Pérez, A., García, S., Gozalez, J., Coello, C., Herrera, F. (2017). An Evolutionary Multiobjective Model and Instance Selection for Support Vector Machines With Pareto-Based Ensembles. IEEE Transactions on Evolutionary Computation, volume (21), pages $863-877$.

Vapnik, V. (2006) Estimations of Dependences Based on Empirical Data. Springer. New York, USA.

Xue, B., Zhang, M., Browne, W., Yao, X. (2016). A Survey on Evolutionary Computation Approaches to Feature Selection. IEEE Transactions on Evolutionary Computation, volume (20), pages 606-626.

Yao, L., Ge, Z. (2017). Locally Weighted Prediction Methods for Latent Factor Analysis with Supervised and Semisupervised Process Data. IEEE Transactions on Automation Science and Engineering, volume (14), pages $126-13$. 